AUTHOR:

Jabulani Nyoni

https://orcid.org/0000-0002-9711-2150

AFFILIATION:

University of South Africa

CORRESPONDENCE TO:

nyonij@unisa.ac.za

DATES:

Published: 25 June 2021

HOW TO CITE THIS ARTICLE:

Nyoni, J., 2021. Criticalities of NonVerbal Reading Competencies: An Afrocentric Ethnological Approach to Qualitative Research. KOERS Bulletin for Christian Scholarship, 86(1). Available at: https://doi.

org/10.19108/KOERS.86.1.2486

COPYRIGHT:

(C) 2021. The Author(s)

Published under the Creative

Commons Attribution License.

\section{Criticalities of Non-Verbal Reading Competencies: An Afrocentric Ethnological Approach to Qualitative Research}

\author{
ABSTRACT \\ Body contact and body language reading are unique and existential and, although culturally \\ dependent and socially embodied, they are critical ethno-specific communication forms \\ confined within contextual geo-spaces. The interactive narratives of ethno-specific, non-verbal \\ communication in my qualitative research approach were facilitated using e-mails, web blogs \\ and thread observations generated by senior research leaders who shared their views on \\ appropriate Afro-ethno-specific qualitative data collection methodologies. Afro-ethno-specific \\ qualitative data collection methodology needs a new narrative that focuses on creating \\ Afrocentric research practices and data collection instruments that are validated for African \\ contexts. Research findings indicate that non-verbal reading competencies that take cognizance \\ of the application of the $3 \mathrm{Cs}$ of non-verbal communication; context, clusters and congruence \\ that are group and Afro-ethno specific were often ignored by researchers in South Africa. The \\ article argues that the application of Afro-ethno specific non-verbal reading competencies, \\ knowledges and skills is critical for it takes into cognizance people's ethnic origin, culture, \\ identity, race, nationality, norms, values, religion or belief systems.
}

Key words: 3 Cs of non-verbal communication, trustworthiness, criticalities, ethno-specific cultural embodiments, Afrocentric non-verbal reading competencies

\section{Introduction}

Irrespective of the area of study or preference for defining data (quantitative, qualitative or mixed methods), accurate data harvesting is crucial and fundamental to maintaining the integrity of research. The selection of appropriate data harvesting instruments (existing, modified or newly developed instruments) and clearly delineated instructions for their correct use reduce the likelihood of errors occurring and increase chances that credibility and trustworthiness may be achieved. The data harnessing component of research is common to all fields of study, including social sciences, the humanities and anthropology. While methods vary according to discipline, the emphasis on ensuring accurate and honest harnessing remains the same. Fit-for-purpose data harnessing methods minimise inaccuracies inherent in inappropriate data gathering processes and maintain ethical observations and the integrity of the research.

In qualitative research, a wide range of tools might be used to explore the deeper meanings of participants' responses. For triangulation purposes, the researcher(s) may use several data harnessing tools in order to make sense of participants' accounts of their experiences, voices, views and other body language expressions. In qualitative research, there is quite several data harnessing methods, including observation, diarising, journaling, focus groups, chat rooms, bulletin boards and observing body language (nonverbal cues). In the postcolonial African context, data harvesting may not be adequate to justify credible findings if the observed expressions of body language (non-verbal) have not been considered. This article deals with these observed, non-verbal expressions. 
During the colonial era, indigenous peoples were ethnically, tribally and racially segregated, and their lives were contaminated with hatred and suspicion. It takes a lot of time to recover from such injustices and pain. Trust and understanding need to develop before people can heal, and only then can they accept one another and become one in reconciliatory laagers. In South Africa, tribal and other racial communities used different body languages for communication purposes in the political and socioeconomic arenas during the apartheid era. Researchers need to appreciate these dynamics fully when they conduct qualitative research, because the country is experiencing bouts of mistrust around the matter of investigating various social groupings and usages.

According to various researchers, non-verbal communication is thought to account for between 50 per cent and 70 per cent of all communication (Mehrabian \& Wiener, 1967; Mehrabian \& Ferris, 1967. Understanding non-verbal communication is critical, but it is also essential to note that other cues and expressions are ethno-specifically contextualised, clustered and compared, to look at signals as a group rather than focusing on a single action.

Noticing the signals that people give with their non-verbal communication is a very useful social skill. Some researchers can read these signals naturally and some are notoriously oblivious to them. Fortunately, by paying a little extra attention, one can learn to read nonverbal communication and with enough practice it can become second nature.

\section{The statement of the problem}

Many scholars believe that a greater portion of people's everyday communication spaces is non-verbal (Guruvayurappa, 2008); Lee, 2007; Reiman, 2008; Redhead, 2003). Albert Mehrabian, a researcher, breaks down this percentage and details the importance of nonverbal communication channels compared to verbal channels. Two of his research studies (Mehrabian \& Wiener, 1967 and Mehrabian \& Ferris 1967) combined provide the 55/38/7 formula. In the rest of this article, three elements of communication are discussed, namely words, tone and non-verbal communication. The article also looks at the so-called 3 Cs of non-verbal communication and their role in providing an understanding of the relationship between non-verbal communication and data harnessing in ethno-specific communication spaces in the Afro-specific contexts.

The three above-mentioned elements are harnessed simultaneously to send an overall message. Tone of voice and non-verbal communication are often the most powerful elements of communication. However, non-verbal communication is also used on its own and is thought to be one the most telling modes of communication. In his research, Mehrabian (1972) supposes that, proportionally, the three elements of communication are not of equal importance. He claims that non-verbal communication conveys most of the information shared when people communicate face to face:

- Words (the literal meaning) account for $7 \%$ of the overall message.

- Tone of voice accounts for $38 \%$ of the overall message.

- Body language accounts for $55 \%$ of the overall message.

The following phenomenological question was posed to ten senior qualitative research experts in order to explore the thoroughness and awareness of data harvesting methods used to obtain data on ethno-specific, non-verbal communications methods from an ethnospecific group of qualitative research participants involved in the Black 500 Schools Project in five South African provinces.

How do researchers in South Africa take into cognizance the use of ethno-specific nonverbal reading competencies during the process of conducting research with indigenous people in order to reach believable and credible insightful findings? 


\section{Theoretical framework}

The multiple subjectively felt meanings and relationships between body, voice and psyche have in recent years been the subject of renewed attention in education, psychology, philosophy, body sociology as well as qualitative research in education (Csordas, 1999; Køppe, 2004; Petzhold, 2006; Koch, 2006; Merleau-Ponty, 1962/2004). Despite many different theoretical views, there is today a large degree of agreement that the body, voice and psyche, as well as the individual's relationship with the surrounding world, must be regarded as a cohesive dynamic and an organic unit. The surrounding world is composed in relation to the 3 Cs (context, clusters and congruence). The written text is based on this primary theoretical view, but the findings are also challenged by criticism from Sparkes (2002:146).

Where bodies have been focused on, they have been heavenly theorized bodies, detached, distant, and for the most part lacking intimate connection to lived experiences of the corporal beings that are the objects of analytical scrutiny (...). Against this backdrop I have asked the question of how we might begin to take more seriously the lived body, the phenomenological and subjective experiences of those involved in research.

Another purpose of this article is to take Sparkes's (2002) criticism seriously and thus to contribute to the development of high-quality qualitative research: to find ways to research into the language of the body and thereby open up new methodological possibilities that would bring us closer to the body's senses, feelings and multidimensional communication in African research contexts. For traditional sciences, non-verbal language is an inaccessible universe.

\section{Qualitative research in context}

Qualitative research is a systematic inquiry into aspects of our world. It is systematic because, whatever the method, the researcher manoeuvres through specific and purposeful steps to design research, collect data and analyse results. Qualitative research is inquiry, because it invariably involves questioning - though definitive answers may be elusive or not even the goal. Qualitative refers to a way of conducting, and conceiving of, research. In qualitative research the researcher is the instrument or the tool for designing research, collecting data and analysing results. Qualitative research, in contrast to quantitative research, generally does not translate aspects of the world into numbers to be analysed mathematically. Instead, it analyses the world through the lenses the researcher brings to bear on the data.

\section{The $3 \mathrm{Cs}$ of non-verbal communication channels clarified}

One way of increasing research accuracy is by applying the 3 Cs of non-verbal communication: context, clusters and congruence. Context includes the environment where the situation plays out, the people's shared history and many other factors, including each person's role (e.g. researcher or participant).

Looking for non-verbal communication gestures in clusters prevents us from allowing a single gesture or movement to define a person's state of mind or emotion. A person who crosses her arms at her chest could be perceived as resistant and close-minded, but if the person's shoulders are raised and her teeth are chattering, she might just be cold or scared.

Finally, congruence means that the words spoken match the speaker's tone of voice and non-verbal communication. When someone falls, but then says she is fine, her words may be contradicted by her facial expression (e.g. a grimace because she is in pain) or tone of voice (e.g. she tries to hold back tears) and you might want to probe a little deeper. 
The 55/38/7 percentage and the 3 Cs of non-verbal communication remind us that a single gesture or comment does not necessarily mean something - we should take note of such a gesture or comment, but we need to observe more in order to get a better understanding of what is going on. Non-verbal communication is the conscious and unconscious movements and postures that communicate attitudes and feelings. Non-verbal communication refers to various non-verbal signals that may reveal a person's unspoken intentions or feelings. These behaviours are often physical and include, but are not limited to, facial expressions, body posture, gestures, eye movement, touch and the use of space.

Facial expressions are among the most universal forms of non-verbal communication. In this article I posit that the interpretations and meanings of facial expressions are not generic but ethno-specific. The expressions used to convey fear, anger, sadness and happiness are not similar throughout the world. Researcher Paul Ekman (2009) has found support for the universality of a variety of facial expressions tied to emotions, including joy, anger, fear, surprise and sadness, but such findings may not be generalised to different races and ethnic or tribal groups. Qualitative researchers need to acquire ethno-specific knowledge and competencies to work with specific Afrocentric groups of people in order to understand their non-verbal forms of communication and to harvest data in conjunction with other Eurocentric methodologies.

\section{Afro ethno-specific competences in African research}

In this article the prefix "ethno-" refers to "people" in context; it is combined with other words to include the meanings "race," "culture" and "people". Culture is "the learned patterns of behaviour and range of beliefs attributed to a specific group that are passed on through generations. People from culturally and linguistically diverse (CALD) backgrounds often require aged care services sensitive to their specific cultural, spiritual, dietary and linguistic needs. It includes ways of life, norms and values, social institutions, and a shared construction of the physical world."

Competence is used to describe behaviours that reflect the appropriate application of knowledge and attitudes. Race and ethnicity are not only physical attributes of people, but also ways of seeing and understanding the world. While the two terms are often used interchangeably, race is a way of classifying individuals and groups based on physical characteristics, particularly one's skin colour. Ethnicity, meanwhile, delineates one's place of origin or nationality, one's cultural background or ancestry, one's language and, by extension, one's belief system.

Despite the concrete physical and sometimes geographical roots tied to specific racial and ethnic identities, it is important to understand that race and ethnicity are also ideologies, or ways of seeing and understanding the world around us. Race and ethnicity are therefore imbued with meaning. They are not only used as descriptors, but also as markers of broader concepts and relationships. Race and ethnicity can mark you as belonging to a group or as an outsider, as different. These markers not only designate one's skin colour or cultural background, but also function in a larger system and in relation to other racial and ethnic identities. In this system, certain groups have more power and privileges than others.

For centuries, ethnic minority communities have battled mightily to secure rights and opportunities that they have systematically been denied. This is a struggle that continues to this day in new and evolving forms.

\section{Non-verbal communication}

Action speaks louder than words. Culture influences non-verbal behaviour and non-verbal communication reading competencies in profound ways. In the context of this study it 
is important to distinguish between gestures and facial expressions. Throughout this article movement of a part of the body intended to suggest a certain meaning or feeling (especially movements of the hands of head) is known as gesture (Hornby, 2002). Facial expressions are the form of kinesics where the face is second only to the eyes in the amount of information it conveys about a person (Rodrigues, 2005). This is to say that the face plays no role in gestures (or only a very small role), but as far as facial expressions are concerned, the face and its most important component, the eyes, are crucial for non-verbal communication. To find out what people feel or believe we look at context and construct an interpretation. However, most forms of nonverbal communication can be interpreted within the framework of the culture and language in which they occur. Cultures differ in specific enacted behaviours, movements, posture, vocal intonations and gestures; even dances are specific to a culture (Lustig \& Koester, 2003).

Reading non-verbal signals is like reading a story. One word does not tell the whole tale, and neither does one isolated gesture. In order to read non-verbal signals successfully, one should consider at least a combination of three gestures from the following list:

\subsection{Facial expression}

Africans try to avoid eye contact as a sign of respect. In rural South Africa, particularly in patriarchal African areas, it is considered rude to make eye contact with anyone who is considered superior. Making eye contact when communicating with a person who is older or of higher status is considered a sign of disrespect or even aggression in many parts of Africa. There is even a Zulu proverb that states: "The eye is the organ of aggression." Since the visual sense is dominant for most people, eye contact is an especially important type of nonverbal communication. The way you look at someone can communicate many things, including interest, affection, hostility or attraction. In most western countries, frequent eye contact is a sign of confidence and attentiveness. Westerners tend to assume that a conversation partner who looks away is either disengaged or lying.

\subsection{Posture}

The way one sits, or stands is key in understanding how one is perceived by others. To project confidence, for instance, one should have one's shoulders back and have one's head held high (but not too high). One should use one's hands to emphasise one's spoken words, as this will also create a more confident persona. For example, African women are expected to wear headgear (doek) or cover their heads when attending social gatherings such as gkotla meetings. Women were denied access to the crucial loci of power, and to the seat of socio-political authority and control the kgotla. African women tend to bend their knees slightly, put their hands together when greeting, and their shoulders should never be exposed but fully covered. Men sit on stools as well as on the ground or on reed mats. However, to a certain extent colonial interference as well as liberal views have forced gradual transformation and genda emancipation.

\subsection{Body movements}

Hands, arms, feet and the head can "do the speaking" in many cases. Crossing the arms, tapping the foot rapidly or putting one's hands on one's hips can all say things that might not be very positive. On the other hand, clapping, tapping one's foot to music or extending a hand to shake can say great things. As a further example of such body movements, Africans shake their head sideways when in disagreement and up and down when in agreement.

\subsection{Arm position}

The crossing of hands across men's chests might signify that something very big is being discussed; this could be a matter of death in family or in the area. It exemplifies emotional and psychological distress. An open hand and a pointing index figure stresses anger, warning and aggression. 


\subsection{Hand gestures}

When trying to communicate despite a language barrier, it is natural to use gestures as a way of illustrating one's point. We assume that such gestures are helpful, since our words are being visually reinforced. Hand motions are, however, culturally relative and the wrong gesture can inadvertently lead to confusion or offence. The thumbs-up sign is seen, in Greece and Sardinia, as equivalent to showing the middle finger. Tapping one's finger to one's temple is a gesture to indicate memory in North-America, but it suggests insanity in Russia.

\subsection{Foot placement}

For instance, African women often sit with their legs crossed, particularly in social gatherings. It is a sign of respect. A man who is a leader in a group may sit on a high stool or chair, while most commoners will sit on the ground next to their knobkerries which are always carried around for self-defence. Only in the modernist and post-modernist eras have leaders taken to arguing with their legs crossed and while sitting on a chair or stool.

\subsection{Stance}

Just as gestures can display dominance, a person adopting a wider and taller stance, while also being relaxed, is another show of dominance. High-status individuals will also take up more physical space to show their dominance and their mastery of the situation. In other words, an expansive pose signals power and achievement.

\subsection{Paralanguage}

Vocal characterisers (such as laughing, crying, yelling, moaning, whining, belching and yawning) all send different messages in different cultures. The "noise" people make also carries meaning. South Africans from different backgrounds, for example, use several sounds that have special significance. In SeTswana, the most common of these is the "hmhm" sound, which can mean "we are in agreement", "I understand," or "continue". In South Africa, the "yo!" sound signifies surprise.

\section{Methodology}

As a critical theorist, I am challenged to translate body experiences into words and written language. I am inspired to find a place where "the soul can sing and the bones dance" (Snowber, 2002:20). I have therefore used phenomenological, narrative and autoethnographic research methods (Snowber, 2002; Merleau-Ponty, 1962/2004; Engel, Rønholt, Svendler Nielsen \& Winther, 2006; Sparkes, 2002, 2003) to harvest data from 10 schools participating in the 500 Schools Project in South Africa.

Use of the 3Cs model of non-verbal communication kept the living, expressive and sensual quality alive in both the research process and its mediation. Phenomenological descriptions captured the experiences, and further kept the phenomenon alive in a process which challenged the researchers' subjective involvement in a critical dialogue between intuition and reflection (Merleau-Ponty, 1962/2004). The interactive narratives of ethno-specific non-verbal communication in qualitative research were captured through deliberative kgotla discourse analysis generated through oral exchanges with ten (10) senior university researchers who shared their views and recounted the kind of data collection methods they used in as part of the 500 Schools Project in five South African provinces, namely Mpumalanga, Free State, Eastern Cape, KwaZulu-Natal and Limpopo. Deliberative kgotla public meetings are purposively designed to capture "authentic" and "unfiltered" opinions of others on a subject. The conversations were held over four sessions and were terminated after data saturation.

Thematic content analysis (TCA) was used to analyse data. TCA investigates the thematic content of chat room conversations (or other texts) by identifying common themes in the 
texts provided for analysis. TCA is the most foundational of qualitative analytic procedures and, in some ways, informs all qualitative methods. In conducting a TCA, the researcher's epistemological stance is objective or objectivistic. A list of common themes was grouped and distilled from the texts to give expression to the communality of voices across the spectrum of participants. Every reasonable attempt was made to employ names for themes drawn from the actual words of participants and to group themes in a manner that directly reflected the texts. While sorting and naming themes required some level of interpretation, "interpretation" as such was kept to a minimum. This researcher's own feelings and thoughts about the themes - or about what the TCA themes signified - were largely irrelevant to the TCA itself.

In Phenomenological Research, an elaborate and articulate set of analogous procedures required that identified meaning units be situated in relation to context and structure for each participant and then for the participants.

\section{Discussion of results, findings, conclusion and recommendations}

The critical, theoretical qualitative research lens applied in conjunction with the 3 Cs model assisted this researcher in exploring the voices of other senior qualitative researchers through texts, conversations, diaries and observations on the collection of data from African participants. Investigation was undertaken into how they took cognizance of the inclusion of non-verbal communication forms and the processes they followed to collect material evidence for their research projects in Africa. Their purpose was to read accurately and interpret appropriately the expressions of participants in the 500 Schools Project in five provinces in South Africa. This researcher also used the same lens in his endeavour to understand how the multiple subjectively felt meanings and relationships between participants' body and psyche were factored in.

It is important to note that non-verbal communication entails the use of body language, facial expressions, gestures, eye contact, posture and tone of voice. These speak the loudest. The ability to read non-verbal communication signals, such as expressions, is a powerful competence that can help researchers harvest material evidence that allows for deeper and more meaningful interpretations and analyses.

Movements and gestures made using hands, arms, legs and other parts of the body and face are the most pervasive types of non-verbal messages and the most difficult to read and control. Humans express attitudes towards themselves and others vividly through body movements and posture. Body movements convey true messages about feelings that cannot be masked. Because such avenues of communication are visual, they travel much farther than spoken words and are unaffected by the presence of noises that interrupt or cancel out speech. Given this understanding, this researcher used thematic content analysis (TCA) in the study and came up with the following themes: Cultural and political isolation conflict awareness, non-verbal communication reading competencies, generalisation of data harvesting methods, political consciousness competencies and decolonial (applying African engineered research methodologies) research practices.

\section{Cultural and political isolation conflict awareness}

Every culture interprets non-verbal cues such as gestures, posture and carriage, vocal noises (like soft tones and groaning) and degree of eye contact differently. Afro ethno-specific nonverbal forms of communication are uniquely contextualised, clustered and congruent to a cultural community and may not be generalised or replicated anywhere. In this study context, the cultural community investigated was indigenous people of South Africa. 
Ntuli (2012) writes:

[A]spects of intercultural communication illustrate how a lack of understanding of other people's culture and non-verbal behaviours can cause misunderstandings and even conflict.

This researcher agrees with the author quoted above, particularly with reference to postcolonial multicultural societies like South Africa. Relationships which have predominately been polarised on racial grounds and political isolation for centuries in South Africa tend to hamper research endeavours as different racial groups become suspicious and distrustful of one another. Previously, power relations in South Africa were vertically inclined and white supremacists were in total control; the black majority played second fiddle to the ruling white minority. Under such circumstances, researchers need to apply extreme caution and take ethical approaches when dealing with different ethnic groups and other races during research undertakings. It would have been naïve to use Eurocentric data collection instruments that had never been scientifically validated for research among indigenous South Africans who experienced colonialism. Most researchers seemed favour focus groups, structured interviews and observations, among others, but not even one researcher used the non-verbal data harvesting method. In the process of collecting data, I was consciously guided by the auto-ethnographic method. According to Maréchal (2010:43) "auto-ethnography is a form or method of research that involves self-observation and reflexive investigation in the context of ethnographic fieldwork and writing". Most of the participants' narratives of data collection methods and instruments might be summed up as follows:

In our 500 Schools Project, we used focus groups, observations, diaries and structured interviews. Our research groups were mostly multicultural. We relied on material evidences that were collected through the methods that were also generally suggested in the research proposal.

When pressed further to explain the reasons why non-verbal forms of communication were not considered, the composite response was:

Multi-ethnic, multicultural research acknowledges differing cultural frameworks and intentionally draws those cultural perspectives into processes of research approaches using conventional, well-grounded methodologies.

How can data collection instruments coined in Western countries be appropriate for ethnicities in Africa without being validated to ascertain their suitability? Culture represents the environment in which a person is brought up in and in which one learns appropriate behaviours, norms, values and beliefs. This environment plays such a critical part in the development of a person's personality that scientists, to this day, are still arguing over whether it (environment) or a person's genetic make-up (biology) plays a larger role in the formation of personality. Every culture has different levels of physical closeness that are appropriate in different types of relationship, and individuals learn these distances from the society in which they grow up.

Body movements can be used to reinforce or emphasise what a person is saying and offer information about the emotions and attitudes of a person. However, it is also possible for body movements to be inconsistent with what is said. A skilled ethno-specific non-verbal reading competencies observer may be able to detect such discrepancies in behaviour and use them as a clue to a person's real feelings. 


\section{Non-verbal communication reading competencies}

Ethno-specific non-verbal communication reading competence is a complex activity that combines several abilities and incorporates both cross-cultural expertise and body language skills. For the purposes of this study, the term refers to the content and form of non-verbal communication among people of different cultures. Competence in ethnospecific communication is the ability to take part effectively in a given social context by understanding what is being communicated and by employing appropriate deportment, behaviour, cues or signals to convey an intended message.

When either the tone or the non-verbal aspects are not present, or if they are incorrectly interpreted, the emotional meaning of the words can be lost, leading to an inappropriate response by the listener. Theorist Campinha-Bacote, (2007) developed the Culturally Competent Model of Care, which identifies five constructs of cultural competence: cultural awareness (a self-reflection of one's own biases), cultural knowledge (obtaining information about different cultures), cultural skills (conducting an assessment of cultural data of participants), cultural encounters (personal experiences with participants of different backgrounds) and cultural desire (the process of wanting to be culturally competent). Asked to comment about their responses to some of the non-verbal expressions displayed by participants in the field, researchers typically answered as follows:

I used to think that anyone doing something weird was weird. Now I know that it is the people who call others weird who are weird. It's true. Our judgments and personal biases (embedded in our mental maps) are the weirdest thing about us. They are also the biggest impediment to cross-cultural listening.

The ability to read nonverbal signals with understanding can increase trust, clarity, and add interest to one's data collection in research when yielded properly. Learning how to become more sensitive to body language and nonverbal cues will make one a better researcher.

\section{Generalisation of data harvesting methods}

Within professional, ethical qualitative research praxis, a major trend globally has been a move towards attempting to restrict traditional research methodologies because of the mistaken belief that projective methods constitute pseudoscience, that they are devoid of legitimate scientific support, and that they might be harmful to the client (see Lilienfeld, Lynn, and Lohr, 2003). There is also a lack of clarity about the appropriateness of such studies involving ethnic minorities and non-Americans (Wood, Garb, Lilienfeld \& Nezworski, 2002). Generalisation of a construct to a new ethnic group - or any other group - may not be a prudent scientific justification for validation processes and is by nature a lengthy exercise. What this researcher found both intriguing and ambivalent the mistaken belief that the methodologies that work in the Western world necessarily apply on other continents. For example, the data collection methods used in the study were focus group discussions, structured interviews and observations. On the question of what data collection methods were suggested in the research proposal, one of the critical responses read:

It was suggested that we should use focus group discussions, structured interviews and observations. We acted as facilitators. The principals and research coordinators organised parents for us and we went in to do our research.

There was no consideration of scientific and methodological problems related to culture, identities, race, ethnicity and political struggle awareness. There was no examination of epistemological considerations as they relate to the study of racial and ethnic groups. Fundamentally, the research methodology and procedures suited Eurocentric praxis and was therefore were biased against African contexts. Non-verbal communications may not be generalised as other embedded cultural and linguistic characteristics may embody 
differentiated deeper meaning and only a researcher who is competent in reading ethnospecific cues can accurately make informed inferences and conclusions.

\section{Political power consciousness competencies}

Consciousness, viewed within this narrative, indicates people's increasing political understanding and their willingness to participate in political action. Political awareness is understood as political knowledge, whereas consciousness indicates the commitment to resist the state with a view to transforming existing political and economic realities. Consciousness, therefore, is not considered to be increasing political awareness, but instead viewed as applied awareness.

If it is said, for example, that someone is politically correct, it means that the person is extremely careful not to offend or upset any group of people in society who are at a disadvantage, or who have been treated differently because of their sex, race or disability.

As a researcher, one puts human rights, social justice and peace at the forefront of one's practice, and cultural competence and socio-political consciousness are the foundation of one's work. Competencies are the abilities to use knowledge and other capabilities necessary for the successful and efficient accomplishment of an appointed task, transaction of work, goal realisation or performance of a certain role in the research process. In the same vein, there are political power relations among people of the same ethnic group. In the African context, women are not as highly regarded or placed on the same pedestal as their male counterparts. Males have their own hierarchical political strata, for they are also placed at different levels depending on the power they hold.

\section{Decolonial research practices}

Postcolonial Studies intends to deconstruct the Eurocentrism that underlies a large part of theory-building in the West. However, it not only challenges Eurocentric claims of universality, but also the complementary assertions of global particularism and cultural relativism (Nyoni, 2013).

Ethical, social and epistemological assumptions and values inform participatory action research from the perspective of decolonisation of methodologies (Battiste, 2001; Sandoval, 2000; González y González \& Lincoln, 2006; Sikes, 2006). Eurocentrism and colonialism continue to be prevalent, even in many liberatory, participatory action research projects. The dismantling of the hierarchical power relationships between participants from the "institutions of higher learning" and participants from the "community" remains a major site of struggle in the process of ethical research.

Salway, Allmark, Barley, Higginbottom, Gerrish and Ellison (2009b:3) point to the way in which:

Unauthorized or insensitive inclusion of data on ethnic groups can lead to negative consequences including: the creation/perpetuation of damaging stereotypes; exaggeration of differences between "groups"; and the production of culturalist explanations that ignore socioeconomic and political factors.

There was deliberate engagement between the principal researchers (participants) and this researcher on the fundamental question of taking into cognizance issues of, among others, the existence of the lack of trust between black and white caused by racial tension that was the norm during the apartheid era. One researcher indicated that: 
My concern has always been that often colonialism is seen and perceived as history, left behind in the dusty closet of the past. Due to this (convenient) understanding people in/from privileged societal, racial, gender positions seem to be unable and, therefore, unwilling to acknowledge the existence of ongoing injustices and systemic inequalities in the world and institutional power structures.

\section{Recommendations}

African scholars must begin to decolonialise research praxis and practices by engaging in critical thought to create appropriate Afrocentric validated research epistemologies and ontologies. They should not rely on methodologies that are created elsewhere and are, in most cases, entrenching a colonial mentality and hegemony. Human rights, ethics and social justice require that researchers need to apply the 3 Cs of non-verbal communication: context, clusters and congruence when doing research in any given community in a society.

\section{Conclusion}

Afro-ethno-specific qualitative data collection methodology needs a new narrative that will focus on creating Afrocentric research practices and data collection instruments that are validated for African contexts. Research findings indicate that non-reading competencies and cues that are group and ethno-specific are often ignored by researchers in South Africa due to cultural, linguistic and racial inadequacies. A competent ethno-specific body language reader does not necessarily know all the rules of the other culture but must be in a position to analyse and interpret non-verbal expressions with a degree of accuracy and certainty. Successful communication depends on getting to know people as individuals, asking questions and seeking to understand their perspective before drawing conclusions about their attitudes and intentions. Testimonies within research findings attest that it is a fallacy to believe that data collection instruments conceived in Western countries are suitable for African contexts. Findings show that researchers concentrate on non-verbal words (the literal meaning), which account for only $7 \%$ of the overall message. The major elements - such as the tone of voice, which accounts for $38 \%$ of the message, and body language, which accounts for $55 \%$ of the overall message - are completely ignored.

\section{References}

Ekman, Paul. 2009. In Clancy W. Martin ed. Lie Catching and Micro Expressions: The Philosophy of Deception, Oxford University Press. https://doi.org/10.1093/acprof:oso/9780195327939.003.0008.

Battiste, Marie. 2001. Decolonizing research: The quest for ethical guidelines for research involving Indigenous populations. In G. Alfredsson and M. Stavropoulou, eds., Justice pending: Indigenous Peoples and other good causes. Essays in honour of Erica-Irene Daes (33-44). Raoul Wallenburg Institute of Human Rights and Humanitarian Law, Lund University.

Campinha-Bacote, Josepha. 2007. The process of cultural competence in the delivery of healthcare services: The journey continues (5th Ed.). Cincinnati, OH: Transcultural C.A.R.E. Associates.

Csordas, Thomas, J. 1999. Embodiment and cultural phenomenology. In Gail Weiss and Honi Fern Haber, eds., Perspectives on embodiment (pp.143-165). New York: Routledge.

Collins, Andrew. 2003. Gestures, non-verbal communication and behaviour. New York: DKC.

Engel, Lis, E., Rønholt, Helle, Svendler, Charlotte, N. and Winther, Helle, eds. 2006. Bevoegelsens poetik. København: Museum Tusculanum.

González y González, Elsa. M. and Lincoln, Yvonna, S. 2006. Decolonizing qualitative research: Nontraditional reporting forms in the academy. Forum: Qualitative social research, 7(4/1). Retrieved April 2, 2010, from http://www.qualitative-research.net/fqs-texte/4-06/06-4-1-e.pdf

Guruvayurappa, Krishna, R. 2008. The basics of non-verbal communication. Retrieved from www. chillibreeze.com [Accessed: 30th June 2014]. 
Hall, Edward, T. 1963. A system for the notation of proxemic behaviour. American Anthropologist, 65(5):1003-1026. https://doi.org/10.1525/aa.1963.65.5.02a00020.

Hall, Edward, T. 1966. The Hidden Dimension. Anchor Books.

Hornby, Albert, S. 2002. Oxford Advanced Learner's Dictionary, $6^{\text {th }}$ ed. In Sally Wehmeier, ed., English Language Dictionaries. Oxford University Press.

Koch, Sabine, C. 2006. Interdisciplinary embodiment approaches. Implications for creative art therapies. In Sabine C. Koch and Iris Bräuninger, eds., Advances in dance/movement therapy. Theoretical perspectives and empirical findings (pp.17-29). Berlin: Logos.

Køppe, Simon. 2004. Kroppens historie In Simo Køppe, Birgit Bork Mathiesen, Jesper Brøsted Sørensen, Bjarne Jacobsen, Mette Skovgaard Væver, Susanne Harder and Susanne Lunn, eds., Kroppen i psyken (pp.15-48). København: Hans Reitzels Forlag.

Lilienfeld, Scott. O., Lynn, Stephen. J. \& Lohr, Jeffrey, M. 2003. Science and pseudoscience in clinical psychology: Initial thoughts, reflections, and considerations. In S. O. Lilienfeld, S. J. Lynn and J. M. Lohr, eds., Science and pseudoscience in clinical psychology (pp. 1- 14). New York: Guilford Press.

Lustig, Myron W. \& Koester, Jolene. 2003. Intercultural competence: Interpersonal communication across cultures. Boston: Pearson Education.

Maréchal, Garance. 2010. Autoethnography. In A. J. Mills, G. Durepos and E. Wiebe, eds., Encyclopedia of case study research (2, pp.43-45). Thousand Oaks, CA: Sage Publications

Mehrabian, Albert. 1972. Non-verbal Communication. New Brunswick: Aldine Transaction.

Mehrabian, Albert \& Wiener, Morton. 1967. Decoding of inconsistent communications, Journal of Personality and Social Psychology, 30 (6):109-114 https://doi.org/10.1037/h0024532.

Mehrabian, Albert \& Ferris, Susan, R. 1967. Inference of Attitudes from Nonverbal Communication in Two Channels, Journal of Consulting Psychology, 31 (3):48-258 https://doi.org/10.1037/h0024648.

Merleau-Ponty, Maurice. 2004. Phenomenology of perception. London: Routledge Classics.

Merleau-Ponty, Maurice. 1962. Phenomenology of perception. London: Routledge Classics.

Ntuli, Cynthia, D. 2012. Intercultural Misunderstanding in South Africa: An Analysis of Non-verbal Communication Behaviour in Context. Intercultural Communication Studies XXI.21(2):20-36.

Nyoni, Jabulani, 2013. Decolonial Multicultural Education in Post-Apartheid South Africa. International Journal for Innovation Education and Research, 1 (3), 83-92. https://doi.org/10.31686/ijier.vol1.iss3.118

Redhead, Philip. 2003. "How to read body language." Retrieved from http://www.empower.tv/esd/ HowToReadBodyLanguage.pdf [Accessed: 10th May 2014].

Reiman, Tonya. 2008. Body language. Retrieved from www.tonyareiman.com [Accessed: 11th January 2015].

Rodrigues, Lawrence. 2005. Body language and facial expressions for flirting, dating and relationships. Retrieved from http://www.learnbodylanguage.org/face_expressions.html_[Accessed: 4 March 2015].

Salway Sarah, Allmark Peter, Barley Ruth, Higginbottom, Gina, Gerrish Kate \& Ellison, George. 2009b. Researching ethnic inequalities, Social Research Update, 58, 1-4, University of Surrey, Guildford.

Sandoval, Chela. 2000. Methodology of the oppressed. Minnesota: University of Minnesota Press.

Sikes, Pat. 2006. "Decolonizing research and methodologies: Indigenous peoples and crosscultural contexts. Review essay." Pedagogy, Culture and Society, 14 (3):349-358. https://doi. org/10.1080/14681360600892017.

Snowber, Celeste, N. 2002. Bodydance: Fleshing soulful inquiry through improvisation. In Carl Bagley and Mary Beth Cancienne, eds. Dancing the data (pp.20-33). New York: Peter Lang.

Sparkes, Andrew. 2002. Telling tales in sport and physical activity. A qualitative journey. Leeds: Human Kinetics.

Sparkes, Andrew. 2003. Bodies, identities, selves: Autoethnographic fragments and reflections. In Jim Denison and Pirkko Markula, eds., Moving writing: Crafting movement in sport and research (pp.51-76). New York: Peter Lang.

Wood, James, M., Lilienfeld, Scot, O., Garb, Howard, N. and Nezworski, Teresa, M. 2000. “The Rorschach Test in clinical diagnosis: A critical review, with a backward look at Garfield (1947)." Journal of Clinical Psychology, 56 (6):395-430. https://doi.org/10.1002/(SICI)1097-4679(200003)56:3<395::AIDJCLP15>3.0.CO;2-O. 